\title{
Neuromodulation of the lingual nerve: a novel technique
}

\author{
Christopher E. Talbot, DO,, Kevin Zhao, DO,, Max Ward, BS, ${ }^{2}$ Aron Kandinov, MD, ${ }^{2,3}$ \\ Antonios Mammis, MD, 1,2 and Boris Paskhover, MD2,3
}

\begin{abstract}
Departments of ${ }^{1}$ Neurological Surgery and ${ }^{3}$ Otolaryngology-Head \& Neck Surgery, ${ }^{2}$ Rutgers New Jersey Medical School, Newark, New Jersey
\end{abstract}

\begin{abstract}
Acute injury of the trigeminal nerve or its branches can result in posttraumatic trigeminal neuropathy (PTTN). Affected patients suffer from chronic debilitating symptoms long after they have recovered from the inciting trauma. Symptoms vary but usually consist of paresthesia, allodynia, dysesthesia, hyperalgesia, or a combination of these symptoms. PTTN of the trigeminal nerve can result from a variety of traumas, including iatrogenic injury from various dental and maxillofacial procedures. Treatments include medications, pulsed radiofrequency modulation, and microsurgical repair. Although trigeminal nerve stimulation has been reported for trigeminal neuropathy, V3 implantation is often avoided because of an elevated migration risk secondary to mandibular motion, and lingual nerve implantation has not been documented. Here, the authors report on a patient who suffered from refractory PTTN despite multiple alternative treatments. He elected to undergo novel placement of a lingual nerve stimulator for neuromodulation therapy. To the best of the authors' knowledge, this is the first documented case of lingual nerve stimulator implantation for lingual neuropathy, a technique for potentially reducing the risk of electrode migration.
\end{abstract}

https://thejns.org/doi/abs/10.3171/2020.2.JNS193109

KEYWORDS neuromodulation; lingual; trigeminal; stimulation; burning mouth; face pain; surgical technique; peripheral nerve

1 CUTE injury of the trigeminal nerve or its branches can result in posttraumatic trigeminal neuropathy (PTTN), sometimes referred to as "trigeminal nerve sensory neuropathy." Affected patients suffer from chronic debilitating symptoms long after they have recovered from the inciting trauma. Symptoms vary but usually consist of paresthesia, allodynia, dysesthesia, hyperalgesia, or a combination of these clinical symptoms. ${ }^{1,2}$

PTTN of the trigeminal nerve can result from a variety of traumas, including iatrogenic injury from various dental and maxillofacial procedures. Lingual pain and paresthesia have also been reported in patients with traumatic basilar skull fracture involving the foramen ovale. ${ }^{3}$ Surgery of the mandibular third molar is the most common iatrogenic injury, with a reported frequency ranging from $0.5 \%$ to $0.6 \% .^{1,4,5}$ Sagittal split mandibular ramus osteotomy, implant surgery, endodontic treatment, and various oral procedures can also injure the lingual nerve.,4

Lingual and/or inferior alveolar nerve injury is a known risk of many oral and maxillofacial surgeries, and while the vast majority $(>90 \%)$ of cases are temporary, those lasting more than 6 months are deemed permanent. ${ }^{6-8}$ The severity of symptoms can vary; however, up to $70 \%$ of patients with PTTN may report pain significantly impair- ing speech or eating. ${ }^{2}$ Thus, obtaining adequate symptom relief in these patients is of the utmost importance in improving their quality of life. Although trigeminal nerve stimulation has been reported for trigeminal neuropathy, V3 implantation is often avoided because of an elevated migration risk secondary to mandibular motion, and lingual nerve implantation has not been documented., ${ }^{9} 10$ To the best of our knowledge, we present the first documented case of lingual nerve stimulator implantation for lingual neuropathy, and a technique for potentially reducing the risk of electrode migration. This novel procedure required a multidisciplinary approach between otolaryngology (ear, nose, and throat [ENT] surgery) and neurosurgery for their respective familiarity with transoral surgery and neuromodulation.

\section{Case Report}

History and Physical Examination

A 58-year-old man was referred to the functional neurosurgery and otolaryngology clinic for tongue pain, numbness, and dysesthesia refractory to medical management. He reported a prolonged course of pain in his tongue and along the left anterior floor of the mouth up to the lingual 

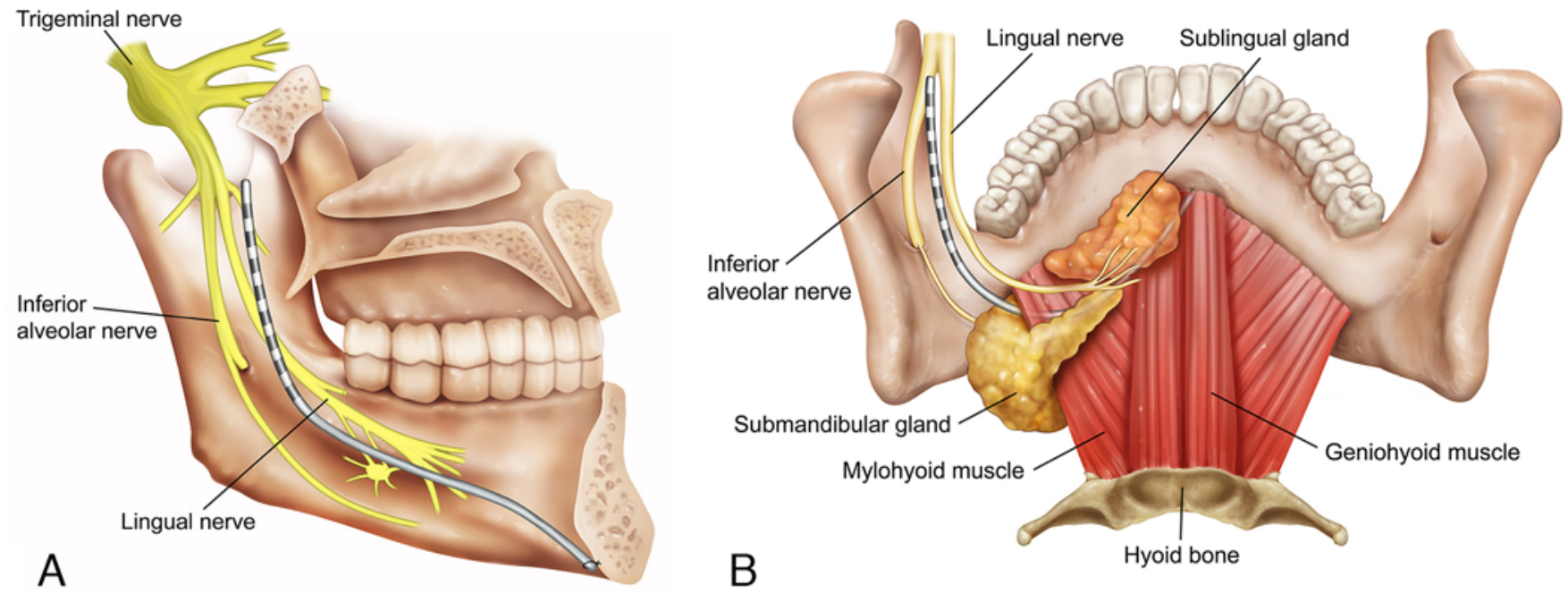

FIG. 1. Illustrations showing electrode placement from medial (A) and posterior (B) perspectives with labeling of anatomically significant structures. Copyright Boris Paskhover. Published with permission.

alveolar ridge. The pain was exacerbated by talking and stress but decreased with mastication. These symptoms began after genioglossus advancement with lingual frenulum release 7 years earlier. At presentation, the patient had already been evaluated by multiple neurosurgeons, interventional pain specialists, otolaryngologists, oral surgeons, and dental practitioners without significant symptom relief. He had undergone pulsed radiofrequency ablation along the left ascending ramus, which improved his symptoms transiently for 2 days. He had received multiple local anesthetic injections targeting the lingual nerve with only temporary relief. He was not a candidate for nerve resection and reconstruction since he was out of the optimal time frame. ${ }^{11}$

He subsequently underwent transoral partial ablation of the left lingual nerve, which resulted in mildly increased numbness and transient incisional pain but no decrease in neuropathic pain. The symptoms from this mild ablation dissipated over the following 2 months. Seeking more aggressive treatment, the patient underwent repeat radiofrequency ablation without significant relief but with increased numbness. For persistent symptoms and severe discomfort refractory to maximal noninvasive and invasive therapies, the patient was deemed a candidate for a novel approach to nerve stimulation, which was performed in two stages. The patient had not undergone any previous attempts at trigeminal nerve or cervical spinal cord neuromodulation.

Gross examination of the tongue and oral cavity was unremarkable. Spasms of the intrinsic tongue muscles were not reported by the patient or observed. There were no motor deficits of the tongue or muscles of mastication. Sensory examination was significant for limited numbness over the left anterolateral margin of the tongue and accompanied by a surrounding region of dysesthesia.

\section{Operative Technique}

To begin, the ENT surgeon performed a careful transoral dissection to the lingual nerve for identification and assistance in appropriate lead placement via a submental approach (Video 1).

VIDEO 1. Intraoperative video clip of a transoral exposure using an endoscope demonstrating exposure of the left lingual nerve before and after placement of a stimulator electrode. Copyright Rutgers-

NJMS, Newark, NJ. Published with permission. Click here to view.

A 2-cm incision was made in the mucosa along the medial aspect of the left mandibular ramus. With the aid of a rigid endoscope to improve visualization, careful soft-tissue dissection was continued toward bone. The periosteum was vertically incised, and the ascending ramus was followed rostrally in a subperiosteal plane until the inferior alveolar nerve was identified entering the inferior alveolar canal. This nerve was traced proximally until its intersection with the lingual nerve was identified. The lingual nerve was carefully isolated from surrounding soft tissue, and scar tissue near the nerve, from prior ablations, was removed. The nerve's course was traced along the floor of the mouth to confirm continuity and integrity, and no neuromas or lesions were identified along this path. The dissection was then carried $2-3 \mathrm{~cm}$ rostral to the inferior alveolar branch point, toward the foramen ovale, to identify a portion of the nerve not previously manipulated, for optimal electrode contact.

A 1.5-cm submental incision along a natural skin crease was made, and the dissection was continued along the inferior edge of the mandible in a subperiosteal plane toward the left floor of the mouth. The dissection was advanced below the mylohyoid musculature so that its attachments would not be disrupted. A tunneling sheath was then placed with endoscopic assistance, and a linear-array percutaneous lead (Medtronic) was introduced into the dissected pocket (Fig. 1). The lead was pushed into place while palpating its path along the floor of the mouth. The stimulator was situated posterior and parallel to the vertical segment of the lingual nerve, and electrodes were confirmed to be in contact with the nerve under endoscopic visualization (Fig. 2). Contacts of the stimulator were confirmed cephalically with contacts along the main branch of V3. 


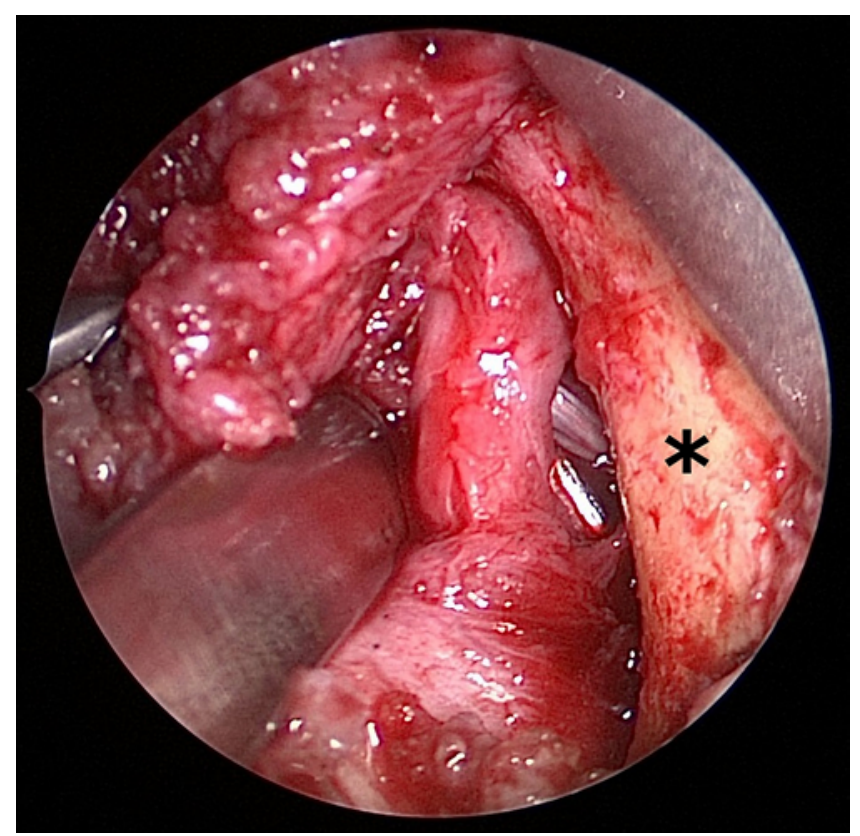

FIG. 2. Endoscopic visualization of the stimulator electrode alongside the left lingual nerve. The left medial mandibular cortex is marked with an asterisk.

The electrode was then anchored to the submental periosteum to permit the electrode to move as a single unit with the mandible in an effort to minimize the risk of electrode migration. Electrode wires were then tunneled from the submental incision to an infraclavicular pocket. Extension wires were attached and externalized in anticipation of a 1-week trial.

\section{Postoperative Course}

Postoperative stimulator activation resulted in instantaneous symptom relief. More superior electrode stimulation resulted in ipsilateral temporalis and masseter spasm suggesting proximal contact with a portion of the trigeminal nerve with motor control. The programming was adjusted to stimulate the inferior alveolar nerve and lingual nerve and ultimately isolate only the lingual nerve distribution. After a trial period of 1 week, the patient returned for the removal of extension wires and implantation of the infraclavicular generator (Medtronic). Stimulation parameters for each of three programs are featured in Fig. 3. At the first postoperative clinic visit, the patient reported a $50 \%-70 \%$ reduction in all symptoms with the stimulator on. His wounds had healed appropriately, and he did not report any discomfort from the tunneled electrode wire. At the 3-month follow-up, he reported 50\%-70\% stable relief of his previous symptoms and was satisfied with this outcome. At the 6-month follow-up, he reported stable relief and was satisfied with this outcome. Plain radiographs show the electrode has not migrated from the desired location (Fig. 4).

\section{Discussion}

Though the vast majority of PTTN cases will resolve,

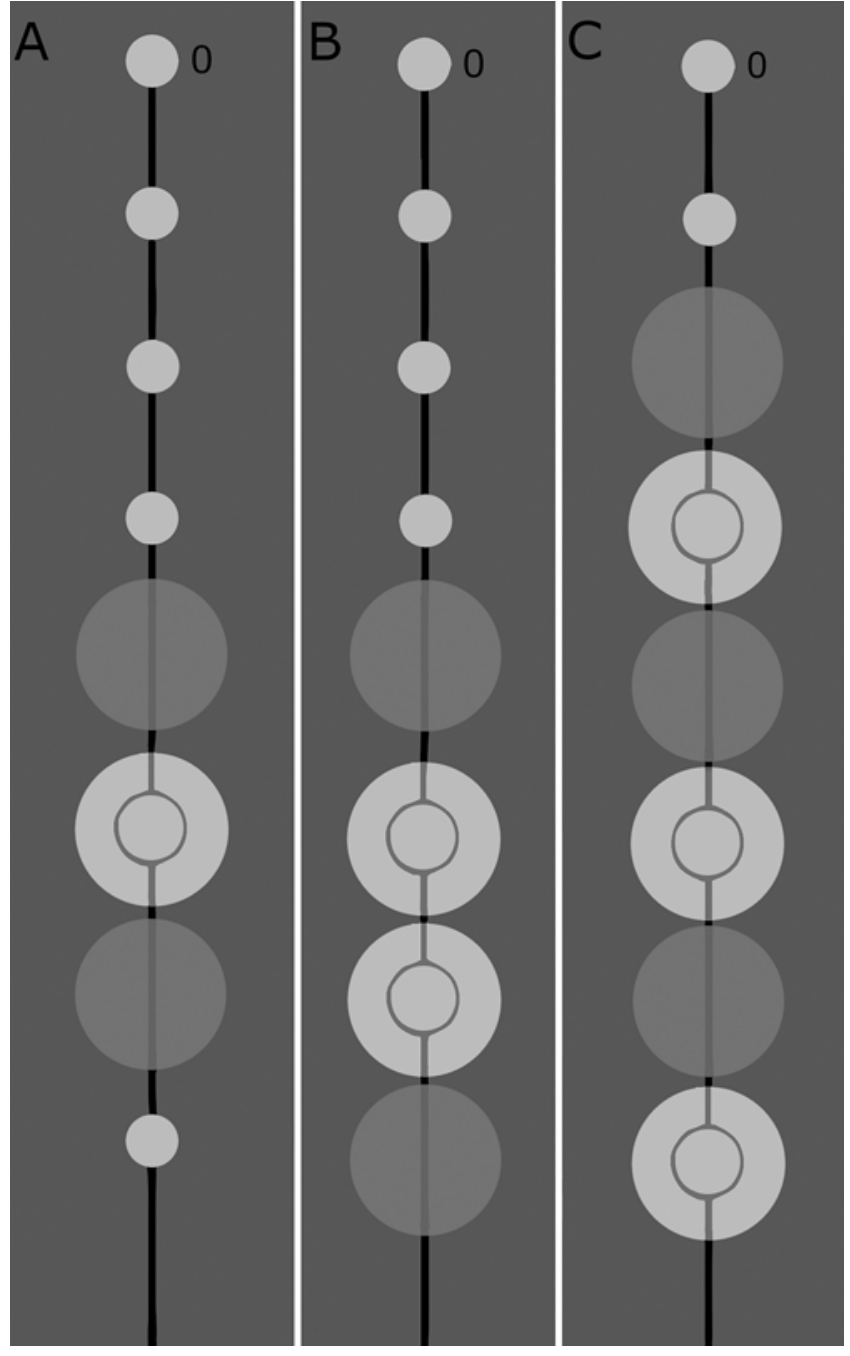

FIG. 3. Stimulator program options. Stimulation parameters for each program were as follows: intensity $2.6 \mathrm{~mA}$, pulse width $280 \mu \mathrm{sec}$, program rate $100 \mathrm{~Hz}(\mathbf{A})$; intensity $3.2 \mathrm{~mA}$, pulse width $220 \mu \mathrm{sec}$, program rate $100 \mathrm{~Hz}(\mathrm{~B})$; and intensity $5.0 \mathrm{~mA}$, pulse width $450 \mu \mathrm{sec}$, program rate $100 \mathrm{~Hz}(\mathbf{C})$.

prompt recognition and repair of iatrogenic nervous injury can help prevent the development of PTTN. Despite this, it is clear that the longer the pain remains unresolved, the less likely it is to completely remit. ${ }^{7}$ Therefore, prompt identification of nerve injury and repair is essential to prevent long-term pain. Surgical repair techniques consist of primary anastomosis, reconstruction with autogenous grafts, and nerve sleeves.? Though primary anastomosis can restore sensation in up to $69 \%$ of patients and function in $41 \%$, it can also cause long-term neuroma formation leading to persistent pain., ${ }^{7,13}$ Nerve and vein grafting is often unnecessary but can be associated with worse outcomes when it is necessary. ${ }^{7,12}$ Nerve sleeves may be a suboptimal option for nervous repair because of poor pain and functional outcomes., ${ }^{7,15}$ Unfortunately, many studies evaluating surgical repair focus on functional recovery and do not provide significant data regarding the longterm pain outcomes of these patients. Regardless, should 

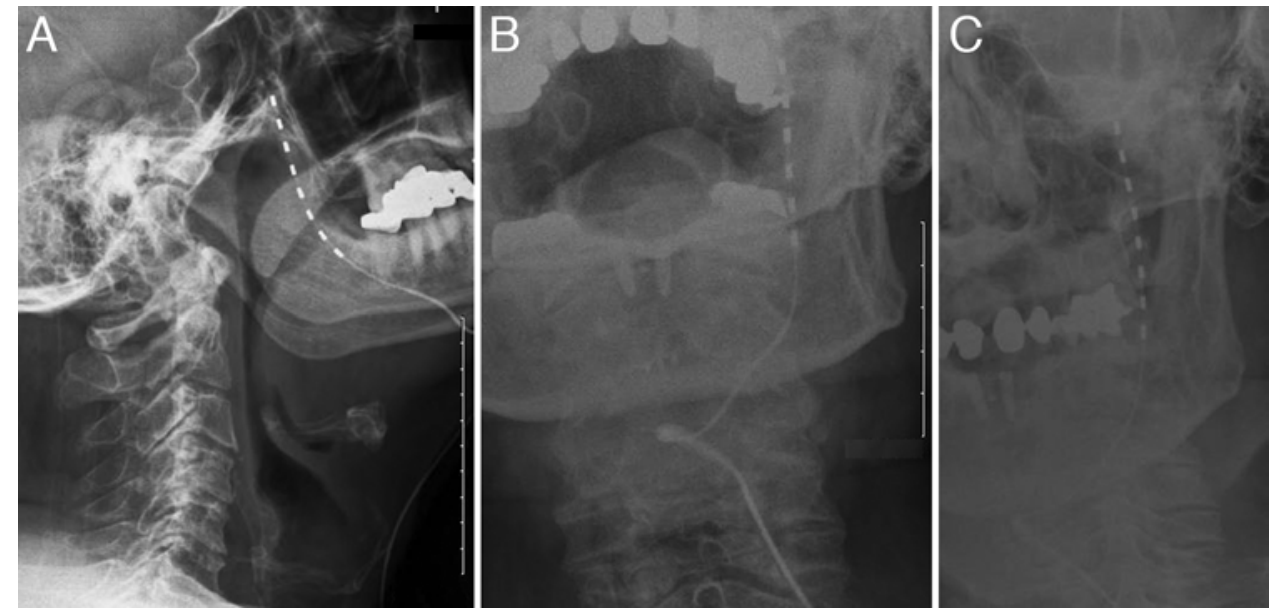

FIG. 4. Postoperative plain radiographs: lateral (A), mouth open (B), and mouth closed (C).

patients develop refractory neuropathic pain, as in our patient, treatment may prove difficult, consisting of multidisciplinary medical, procedural, and surgical management.

In patients with chronic refractory pain, first-line medical therapy often consists of antiepileptics, antidepressants, and standard analgesics, though there are few data to support their use in PTTN. ${ }^{16-18}$ If this therapy fails, patients may undergo nerve blocks, direct nerve ablation, radiofrequency ablation, and low-level laser therapy. Though the latter therapy can improve neurosensory function in lingual PTTN patients, existing evidence may be of low quality and has excluded neuropathic pain patients and pain outcomes. ${ }^{17,19}$ Nerve blocks and radiofrequency ablation may be effective for the management of atypical facial pain; in our case, however, multiple blocks and radiofrequency ablations had failed, with the patient reporting minimal, short-lived relief..$^{20,21}$ Endoscopic trigeminal thermoablation of the inferior alveolar and lingual nerves has been described in the successful treatment of V3 trigeminal neuralgia; in our case, however, ablation failed as well since trigeminal neuropathy is a separate entity. ${ }^{22,23}$

In cases of pain refractory to medications, procedures, and surgery, neuromodulation may be a last resort for satisfactory relief. Though there has never been a documented case of lingual nerve stimulation, Johnson and Burchiel described peripheral stimulation for PTTN and postherpetic neuralgia in $2004 .^{10}$ The 10 patients reported a mean of $58 \%$ pain relief in the study; however, none of the patients received implants for V3 pain as mandibular motion was thought to increase the risk for lead migration or fracture. In 2015, Ellis et al. described 35 patients who were implanted with trigeminal nerve stimulators for refractory craniofacial pain, ${ }^{9} 6$ of whom received implants for trigeminal neuropathic pain; 2 of the 6 patients attained good pain outcomes. However, these treatments only targeted V1 and V2 distributions as the authors feared that an electrode targeting V3 would be prone to migration due to mandibular movement. Hence, while trigeminal nerve stimulation techniques have proven efficacious in the recent literature, they have not been able to treat the lingual nerve distributions of the tongue and oral cavity pain.

We could find no literature on stimulation of the lin- gual nerve, which represents a novel avenue of treatment. Furthermore, we believe that the submental tunneling of a lingual nerve electrode that we describe may be a new method to safely and securely implant the lingual nerve for refractory neuropathic pain. Also, the transoral exposure is essential in ensuring adequate contact with the nerve. In order to optimize electrode placement, it was vital to coordinate with ENT surgeons given their familiarity with the anatomy. This collaboration also helped to avoid several potential pitfalls during a procedure whose risks were heightened given the previously treated state of the areaincluding injury to the submandibular and sublingual salivary ducts, orocutaneous fistula formation, and injury to the lingual and inferior alveolar nerves themselves, among others. The procedure also provided a means of safely minimizing electrode migration risk by tunneling through the floor of the mouth and securing the electrode to the mandibular periosteum. Although there are no data on evaluating the migration risk in V3 implantation, it is important to address this risk, particularly in the case of lingual nerve implantation. It is possible that submental tunneling and anchoring may allow the stimulator to move with the mandible as a single unit, potentially reducing the risk of migration or dislocation, though cadaveric and anatomical studies are necessary to determine if this is true in practice.

Although more data from a larger patient cohort are necessary to better determine the long-term efficacy of this procedure, lingual nerve stimulation provided significant relief to our patient with lingual PTTN, which had failed to resolve after all other available treatments. Peripheral nerve stimulation for the treatment of neuropathic pain is a relatively modern and rapidly evolving field, and we believe that the presented collaboration and method may open several new avenues for the treatment of previously inaccessible trigeminal neuropathies, and similar collaborations may even lead to further innovations elsewhere.

\section{References}

1. Bagheri SC, Meyer RA, Khan HA, et al. Retrospective re- 
view of microsurgical repair of 222 lingual nerve injuries. $J$ Oral Maxillofac Surg. 2010;68(4):715-723.

2. Renton T, Yilmaz Z, Gaballah K. Evaluation of trigeminal nerve injuries in relation to third molar surgery in a prospective patient cohort. Recommendations for prevention. Int $J$ Oral Maxillofac Surg. 2012;41(12):1509-1518.

3. Tekeli KM, Agrawal T, Worrall SF. Unusual case of posttraumatic lingual paraesthesia. Br J Oral Maxillofac Surg. 2008;46(2):157-158.

4. Klazen Y, Van der Cruyssen F, Vranckx M, et al. Iatrogenic trigeminal post-traumatic neuropathy: a retrospective two-year cohort study. Int J Oral Maxillofac Surg. 2018;47(6):789-793.

5. Queral-Godoy E, Figueiredo R, Valmaseda-Castellón E, et al. Frequency and evolution of lingual nerve lesions following lower third molar extraction. J Oral Maxillofac Surg. 2006;64(3):402-407.

6. Blackburn CW. A method of assessment in cases of lingual nerve injury. Br J Oral Maxillofac Surg. 1990;28(4):238-245.

7. Kushnerev E, Yates JM. Evidence-based outcomes following inferior alveolar and lingual nerve injury and repair: a systematic review. J Oral Rehabil. 2015;42(10):786-802.

8. Rood JP, Shehab BA. The radiological prediction of inferior alveolar nerve injury during third molar surgery. $\mathrm{Br} \mathrm{J} \mathrm{Oral}$ Maxillofac Surg. 1990;28(1):20-25.

9. Ellis JA, Mejia Munne JC, Winfree CJ. Trigeminal branch stimulation for the treatment of intractable craniofacial pain. J Neurosurg. 2015;123(1):283-288.

10. Johnson MD, Burchiel KJ. Peripheral stimulation for treatment of trigeminal postherpetic neuralgia and trigeminal posttraumatic neuropathic pain: a pilot study. Neurosurgery. 2004;55(1):135-142.

11. Erakat MS, Chuang SK, Shanti RM, Ziccardi VB. Interval between injury and lingual nerve repair as a prognostic factor for success using type I collagen conduit. J Oral Maxillofac Surg. 2013;71(5):833-838.

12. Cornelius CP, Roser M, Ehrenfeld M. Microneural reconstruction after iatrogenic lesions of the lingual nerve and the inferior alveolar nerve. Critical evaluation [in German]. Mund Kiefer Gesichtschir. 1997;1(4):213-223.

13. Susarla SM, Kaban LB, Donoff RB, Dodson TB. Does early repair of lingual nerve injuries improve functional sensory recovery? J Oral Maxillofac Surg. 2007;65(6):1070-1076.

14. Farole A, Jamal BT. A bioabsorbable collagen nerve cuff (NeuraGen) for repair of lingual and inferior alveolar nerve injuries: a case series. J Oral Maxillofac Surg. 2008;66(10):2058-2062.

15. Pitta MC, Wolford LM, Mehra P, Hopkin J. Use of Gore-Tex tubing as a conduit for inferior alveolar and lingual nerve repair: experience with 6 cases. J Oral Maxillofac Surg. 2001;59(5):493-497.

16. Clark G. Top 60 medications used for orofacial pain treatment. J Calif Dent Assoc. 2008;36(10):747-767.
17. Coulthard P, Kushnerev E, Yates JM, et al. Interventions for iatrogenic inferior alveolar and lingual nerve injury. Cochrane Database Syst Rev. 2014;(4):CD005293.

18. Heir G, Karolchek S, Kalladka M, et al. Use of topical medication in orofacial neuropathic pain: a retrospective study. Oral Surg Oral Med Oral Pathol Oral Radiol Endod. 2008;105(4):466-469.

19. Miloro M, Criddle TR. Does low-level laser therapy affect recovery of lingual and inferior alveolar nerve injuries? $J$ Oral Maxillofac Surg. 2018;76(12):2669-2675.

20. Nader A, Kendall MC, De Oliveria GS, et al. Ultrasoundguided trigeminal nerve block via the pterygopalatine fossa: an effective treatment for trigeminal neuralgia and atypical facial pain. Pain Physician. 2013;16(5):E537-E545.

21. Telischak NA, Heit JJ, Campos LW, et al. Fluoroscopic Carm and CT-guided selective radiofrequency ablation for trigeminal and glossopharyngeal facial pain syndromes. Pain Med. 2018;19(1):130-141.

22. Ashman P, Ward M, Umanoff M, Paskhover B. Minimally invasive trigeminal ablation: novel transoral technique for targeted treatment of V3. World Neurosurg. 2018;118:193196.

23. Tajali Y, Ward M, Abraham M, et al. Minimally invasive trigeminal ablation in patients with refractory trigeminal neuralgia who are ineligible for intracranial intervention. $J$ Clin Neurosci. 2019;70:42-46.

\section{Disclosures}

Dr. Mammis is a consultant for Medtronic, Abbott, Nevro, and Boston Scientific.

\section{Author Contributions}

Conception and design: Paskhover, Talbot, Mammis. Acquisition of data: Paskhover, Talbot, Zhao, Kandinov, Mammis. Analysis and interpretation of data: Paskhover, Talbot, Zhao, Ward, Mammis. Drafting the article: Paskhover, Talbot, Zhao, Ward, Mammis. Critically revising the article: all authors. Reviewed submitted version of manuscript: all authors. Approved the final version of the manuscript on behalf of all authors: Paskhover. Administrative/technical/material support: Paskhover, Talbot, Mammis. Study supervision: Paskhover, Talbot, Mammis.

\section{Supplemental Information Videos}

Video 1. https://vimeo.com/394921612.

\section{Correspondence}

Boris Paskhover: Rutgers New Jersey Medical School, Newark, NJ.borpas@njms.rutgers.edu. 